\title{
Structural Differences in Hippocampal and Prefrontal Gray Matter Volume Support Flexible Context-Dependent Navigation Ability
}

\author{
Thackery I. Brown, ${ }^{1,2,3}$ Andrew S. Whiteman, ${ }^{1,2}$ Irem Aselcioglu, ${ }^{1,2}$ and Chantal E. Stern ${ }^{1,2,3}$ \\ ${ }^{1}$ Department of Psychological and Brain Sciences and ${ }^{2}$ Conte Center for Memory and Brain, Boston University, Boston, Massachusetts 02215 and \\ ${ }^{3}$ Athinoula A. Martinos Center for Biomedical Imaging, Massachusetts General Hospital, Charlestown, Massachusetts 02129
}

\begin{abstract}
Spatial navigation is a fundamental part of daily life. Humans differ in their individual abilities to flexibly navigate their world, and a critical question is how this variability relates to differences in underlying brain structure. Our experiment examined individual differences in the ability to flexibly navigate routes that overlap with, and must be distinguished from, previously learned trajectories. We related differences in flexible navigation performance to differences in brain morphology in healthy young adults using voxel-based morphometry. Our findings provide novel evidence that individual differences in gray matter volume in the hippocampus and dorsolateral prefrontal cortex correlate with our ability rapidly to learn and flexibly navigate routes through our world.
\end{abstract}

\section{Introduction}

The ability to use contextual information and past experiences to guide successful navigation is a critical part of daily life. A fundamental question addressed in the present experiment is how differences in the ability to distinguish between and flexibly navigate overlapping spatial episodes relates to differences in underlying anatomical structure in the healthy human brain.

Learning to navigate routes that share common elements with alternative paths requires the ability to overcome interference from the overlapping representation. The ability to use contextual information to distinguish between or disambiguate overlapping sequential memories (Hasselmo and Eichenbaum, 2005; Zilli and Hasselmo, 2008) depends on hippocampal function in both rodents (Wood et al., 2000; Agster et al., 2002; Smith and Mizumori, 2006; Ginther et al., 2011; MacDonald et al., 2011) and humans (Kumaran and Maguire, 2006; Brown et al., 2010, 2012; Brown and Stern, 2013). Successful navigation of overlapping routes also recruits prefrontal areas (particularly rostral lateral prefrontal and orbitofrontal cortices) and the caudate in humans during both learning (Brown and Stern, 2013) and retrieval (Brown et al., 2010, 2012). Prefrontal-striatal circuitry

\footnotetext{
Received May 23, 2013; revised Dec. 18, 2013; accepted Dec. 20, 2013.

Author contributions: T.I.B. and C.E.S. designed research; T.I.B. performed research; T.I.B., A.S.W., and I.A. analyzed data; T.I.B., A.S.W., and C.E.S. wrote the paper.

This work was supported by an Office of Naval Research Multidisciplinary University Research Initiative grant (ONR MURI N00014-10-1-0936) and a National Institutes of Health Silvio 0. Conte Center for Neuroscience Research Grant (NIH P50 MH094263) to the Cognitive Neuroimaging Lab, Center for Memory and Brain, Boston University. Imaging data were collected at the Athinoula A. Martinos Center for Biomedical Imaging in Charlestown, Massachusetts, which receives support from the NIH National Center for Research Resources Shared Instrumentation Grant Program (NIH P41RR14075).

The authors declare no competing financial interests.

Correspondence should be addressed to Dr. Chantal E. Stern, Center for Memory and Brain, 2 Cummington Mall, Boston University, Boston, MA 02215. E-mail: chantal@bu.edu.

DOI:10.1523/JNEUROSCI.2202-13.2014

Copyright $\odot 2014$ the authors $\quad 0270-6474 / 14 / 342314-07 \$ 15.00 / 0$
}

may be critical for translating context-guided route memory in the hippocampus into flexible selection of alternative navigational responses. Topographical memory and bias toward spatial strategies is associated with hippocampal and prefrontal gray matter structure in humans (Maguire et al., 2006; Bohbot et al., 2007; Hartley and Harlow, 2012; Schinazi et al., 2013), and could further support efficient use of existing route and environment information to avoid navigational errors. In contrast, navigation of completely distinct non-overlapping routes recruits the hippocampus and prefrontal cortex (PFC) less than overlapping routes (Brown et al., 2010, 2012; Brown and Stern, 2013), does not require inhibition of alternative responses, and efficient performance can potentially be supported through learning simple associations and unique stimulus-response pairings. The ability to learn and retrieve distinct non-overlapping routes may therefore benefit minimally from increased neural resources in the hippocampus and PFC.

Together, these functional and structural data led to the prediction that if learning and retrieving overlapping routes is more dependent than learning non-overlapping routes on computations supported by the PFC and hippocampus, then overlapping route navigation during learning would more directly benefit from increased neural resources in these regions. We hypothesized that larger gray matter volumes in the hippocampus and PFC would provide a more robust architecture for representing contextual and spatial details in sequential route memories, and support learning to flexibly navigate overlapping mazes. We tested this prediction in a between-subjects study correlating gray matter volumes in healthy young adults with individual differences in flexible context-dependent navigation during learning. We used a functional magnetic resonance imaging (fMRI) paradigm developed to compare overlapping maze navigation performance during learning with navigation of distinct non-overlapping routes that do not require the use of context to 
a

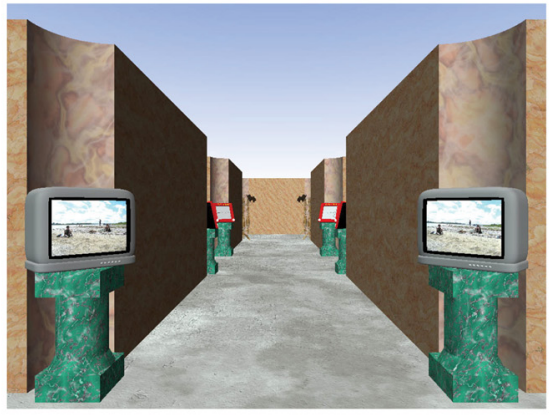

b

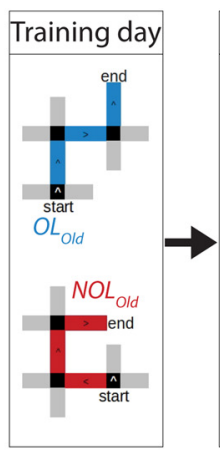

\section{Scanning day}
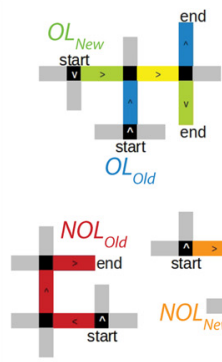

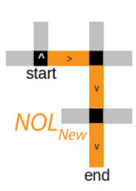

Figure 1. $\quad \boldsymbol{a}$, Example viewpoint during navigation. Unique landmarks distinguish each intersection and facilitate recognition of OL locations. $\boldsymbol{b}$, Examples of overlapping $(\mathrm{OL}$ ) and non-overlapping $(\mathrm{NOL})$ routes. Our study targets performance from novel mazes $\left(0 \mathrm{~L}_{\mathrm{New}^{\prime}}\right.$ in green) that overlap with routes learned during training ( $\mathrm{OL}_{\mathrm{Old}}$, in blue). The $\mathrm{OL}$ hallway is highlighted in yellow. Gray hallways reflect "foil" hallways that are never correct choices. Gray matter correlates of $0 \mathrm{~L}_{\mathrm{New}}$ performance are compared with novel NOL routes $\left(\mathrm{NOL}_{\mathrm{New}}\right)$ shown in orange.

select between alternative navigational behaviors (Brown and Stern, 2013). We predicted people with greater gray matter volume in the hippocampus and functionally related prefrontal regions would have better accuracy on newly encoded overlapping routes and would reach peak accuracy more rapidly across learning trials.

\section{Materials and Methods}

\section{Participants}

Thirty-two participants (20 female; mean age $20.9 \pm 2.9$ years) from the Boston University student community were included in this study. Participants had no history of neurological or psychiatric disorder. Informed consent was obtained in a manner approved by the Partners Human Research Committee and the Boston University Institutional Review Board.

\section{Virtual navigation design and task}

Our experiment targeted learning-period performance on navigating intersections between overlapping mazes, where participants needed to make different context-dependent navigational turn responses at the same landmark/location depending on which route they were currently following. Detailed information about the virtual maze stimuli can be found in our recent fMRI publication introducing this paradigm (Brown and Stern, 2013). Briefly, 20 virtual mazes formed two navigation conditions. Each maze was comprised of three hallways and three intersections. Each intersection contained unique, clearly identifiable objects that served as distinguishing features between the locations (Fig. 1a). Ten mazes were split into five "Overlapping" pairs. Each maze began and ended at distinct, non-overlapping locations, but converged in the middle to share the second hallway with another maze. The unique starting location identified which route was being followed on a given trial. The remaining 10 mazes comprised the "Non-overlapping condition" and were completely distinct from one another.

Participants navigated the virtual routes from a ground-level firstperson perspective. Behavioral accuracies and reaction times were recorded using E-Prime 2.0 (Psychology Software Tools). At each intersection, participants used a button box to select the direction of the next hall in the sequence of locations comprising a maze. Following a correct navigational choice, participants turned down the next hallway and traveled to the subsequent intersection. Incorrect navigational choices were followed with presentation of corrective feedback.

Navigational demands were matched between overlapping and non-overlapping mazes, with the number of left, right, and straight choices counterbalanced across mazes and experimental conditions. Only knowledge of the routes distinguished the overlapping intersections as different from non-overlapping intersections. In non-overlapping mazes, intersections required the same navigational choices in every trial. In contrast, in overlapping mazes the second and third intersections were
On Day 2, participants performed 10 runs of the navigation task $\sim 24 \mathrm{~h}$ after prescan training. During each run, participants navigated once through each of the previously learned and novel mazes. By attending to the feedback for errors, participants learned the 10 new mazes across runs. Mazes belonging to the different experimental conditions were presented in an interleaved manner, with maze order counterbalanced across runs, and run order randomized across participants. Our analysis focused on between-subjects behavioral differences for the new overlapping mazes learned on Day 2.

\section{MRI image acquisition}

Images were acquired at the Athinoula A. Martinos Center for Biomedical Imaging of the Massachusetts General Hospital in Charlestown, MA, using a 3 T Siemens MAGNETOM Trio Tim scanner with a Siemens 32-channel matrix head coil. High-resolution $T_{1}$-weighted multiplanar rapidly acquired gradient echo structural scans were acquired using generalized autocalibrating partially parallel acquisitions $(\mathrm{TR}=2530 \mathrm{~ms}$; $\mathrm{TE}=3.31 \mathrm{~ms}$; flip angle $=7$; slices $=176$; resolution $=1 \mathrm{~mm}$ isotropic $)$.

\section{Voxel-based morphometry}

Gray matter volume was analyzed using standard voxel-based morphometry (VBM) methods in SPM8 (Wellcome Department of Cognitive Neurology, London, UK). Structural images were segmented using SPM8's New Segment option into gray matter, white matter, and CSF images, and bias-corrected. Gray matter segmentation images were spatially normalized into standard Montreal Neurological Institute space using the Diffeomorphic Anatomical Registration Through Exponentiated Lie algebra (DARTEL) algorithm (Ashburner, 2007) for a high degree of intersubject registration. Gray matter images were resampled during normalization $\left(1.5 \mathrm{~mm}^{3}\right.$ isotropic voxels $)$ and spatially smoothed using a $6 \mathrm{~mm}$ full-width at half-maximum Gaussian kernel. As is now standard (Mechelli et al., 2005), VBM analyses were conducted using "modulated" smoothed gray matter images, providing a measure of regional gray matter volume.

\section{VBM statistical analyses}

Behaviorally, we evaluated performance for newly learned overlapping (OL) maze "Critical Decisions" and "counterpart" performance in novel nonoverlapping (NOL) mazes at the second intersection. This allowed us to distinguish gray matter correlates of flexible navigation ability from correlates of navigating NOL routes that do not require the use of context to select between alternative behaviors. Individual subject local gray matter volume estimates were related to Critical Decision performance using two learning-related measures in linear regression analyses in SPM8.

Early task phase performance. Accuracies from individual mazes of each condition were averaged for the first three experimental runs into "Early task phase" performance measures for OL and NOL mazes (as defined in Brown and Stern, 2013). The Early task phase was the period during which learning was greatest for new OL and NOL mazes, and "disambiguation errors" (erroneously making the navigational response 
for the previously learned route) were observed almost exclusively during these early trials, indicating that disambiguation processes are particularly important for accurate behavior during the first three runs. Early task phase accuracy reflects the overall level of performance during early navigation trials, influenced by how well participants are able to (1) overcome interference from previously learned alternative behavioral responses and use existing spatial knowledge from previously learned overlapping routes to rule out turns in new mazes that cannot be correct (OL condition only) and (2) rapidly improve performance from feedback for the second and third trials of each maze (OL and NOL conditions). Participants had equivalent accuracy for the two conditions (mean $(\mathrm{SD}): \mathrm{OL}=0.62(0.174), \mathrm{NOL}=0.63(0.140)$ ). The distribution of scores around the median (Fig. $2 a$ ) indicate substantial behavioral variability for analysis (confirmed by significant structure-performance relationships for both conditions, reported below).

Learning rate. We also analyzed a correlate of Critical Decision learning rate using a signal detection theory-based approach. We limited our analysis to the first six runs (participants reached peak performance by the sixth run/the learning curves reached asymptote). Including additional runs to the model would not add meaningful information to analysis of the learning rate, while including fewer trials might discount learning curve data for some subjects. Taking advantage of nonlinear improvements in individual Critical Choice accuracies, we computed the discriminability or sensitivity index, $d_{a}$, for each subject assuming a standard "unequal variance" dual Gaussian model of target and lure hallway memory strength distributions. This model is used in many fields to estimate receiver operating characteristic curves (e.g., radiology and psychology: Obuchowski, 2003; Wixted and Mickes, 2010), and here is given by Equation 1:

$$
T=\phi\left(a+b \cdot \phi^{-1}(L)\right)
$$

where $T$ is the cumulative target hallway choice rate, $L$ is the cumulative lure hallway choice rate, and $\phi$ is the normal cumulative density function. The parameters $a=\mu_{t} \cdot \sigma_{l} / \sigma_{t}$ and $b=\sigma_{l} / \sigma_{t}$ were estimated via an optimization routine that minimizes the negative log-likelihood of the data given the model. $d_{a}$, was then calculated from these parameters (Eq. 2)

$$
d_{a}=a \cdot \sqrt{\frac{2}{1+b_{2}}} .
$$

Critically, in this design, $d_{a}$ is influenced mainly by the rate mazes are learned. Thus, higher $d_{a}$ values reflect more rapid learning. Participants had comparable $d_{a}$ measures for the two conditions (mean(SD): OL $=$ $1.75(0.750), \mathrm{NOL}=1.95(0.600))$. The distribution of scores around the mean (Fig. $2 b$ ) indicates substantial behavioral variability for analysis (confirmed by significant structure-performance relationships for both conditions reported below). Models were fit and $d_{a}$ was calculated using the statistical software R 2.15.2 (R Development Core Team, 2012).

Individual subject Early task phase performance and $d_{a}$ values were entered as covariates with smoothed gray matter volume estimate images into separate OL and NOL condition second-level multiple regression analyses in SPM8. $T$ statistic images, representing the strength of the linear association, were calculated in SPM. Significant positive relationships indicated local gray matter volume estimates were predicted by Early task phase accuracy or $d_{a}$ measures. Individual subject ages and genders were included as additional covariates for the OL and NOL regression analyses to control for their potentially confounding influence on brain structure and performance.

By separately interrogating the relationship between these factors and brain structure, we were able to examine whether there were any correlates of learning common to both the OL and NOL conditions and directly determine how well volume is predicted by a given factor. However, when differences between conditions were present, it was important to quantify the strength of the specificity of these effects to overlapping maze performance. Therefore, we ran a follow-up analysis with the behavioral measures of both conditions combined into a unified model, and directly contrasted the OL and NOL conditions. Accuracy and $d_{a}$ measures were also correlated within each condition (the average a

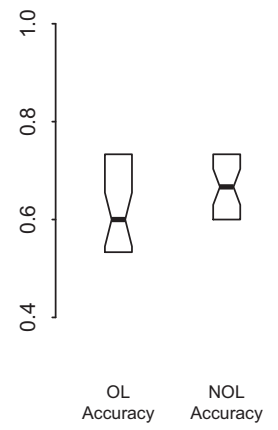

b

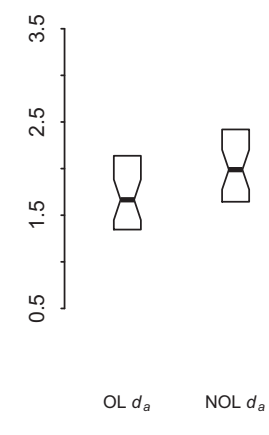

Figure 2. Boxplots of the distribution of $(\boldsymbol{a})$ accuracy and $(\boldsymbol{b})$ learning rate measures for each subject around the median.

level of accuracy is partially influenced by learning rate). By including both behavioral measures from each condition in one unified model, we were also able to determine whether volume differences significantly predicted one behavioral measure while holding variance from the other measure constant.

When considering the results of separate and unified models in our study, it was useful to compare the predictive power of the two approaches. To gain leverage on this question, we refit each model to the VBM data in R, and compared Akaike information criterion (AIC; Akaike, 1974) statistics for each of the separate models against the unified model. The AIC provides a simple measure of expected out-of-sample prediction error for a given model, penalized for the number of parameters, and is used as a relative measure of model quality. A lower AIC value reflects less deviance-indicative of the preferred model. We note that, using this criterion, the separate models may be preferred for between 65 and $85 \%$ of voxels in our search volume within a given behavioral condition. This is not to disregard the unified model, however, which provides a single, comprehensive model for the data facilitating direct comparison of the conditions of interest. To provide a complete understanding of the nature of the structure-performance relationships present in our data, we present results from the unified model alongside our separate interrogations of the behavioral variables.

Based on our strong anatomical predictions from prior functional data (Brown et al., 2010, 2012; Brown and Stern, 2013), and to limit the multiple-comparisons problem, regression analyses were conducted within a restricted region of interest (ROI) volume comprised of the medial temporal lobes (MTL); striatum; and medial, orbital, and lateral PFC. Our search volume was created by combining relevant AAL structural delineations (Tzourio-Mazoyer et al., 2002) from the Wake Forest University (WFU) PickAtlas for SPM (Maldjian et al., 2003, 2004). Analyses were conducted with voxelwise statistical thresholds of $p<0.01$. To limit the occurrence of spurious clusters, we applied a cluster-extent threshold $(k)$ of 168 to maintain a familywise error rate of $p<0.05$, calculated using a 10,000 simulation Monte Carlo analysis in 3dClustSim (for the AFNI software package; http://afni.nimh.nih.gov/afni/). Results were also viewed with an exploratory cluster extent of 100 .

\section{Results}

\section{Hippocampal gray matter volume and early task} phase performance

Local gray matter volume estimates from a large region of the right hippocampus $\left(x y z\right.$ : $38,-25,-11 ; t_{(28)}=3.39 ; p=0.001$; $k=222$ ) had a significant positive relationship with Early task phase performance in the OL condition, indicating people who are better at navigating context-dependent decision points during early trials of overlapping routes have larger hippocampi (Fig. 3). At our exploratory cluster threshold, this positive relationship was also observed in the left hippocampal ROI (xyz: $-28,-28$, $\left.-12 ; t_{(28)}=3.37 ; p=0.001 ; k=109\right)$, and right anterior cingulate cortex $\left(x y z: 14,47,1 ; t_{(28)}=3.48 ; p=0.001 ; k=120\right)$. The 

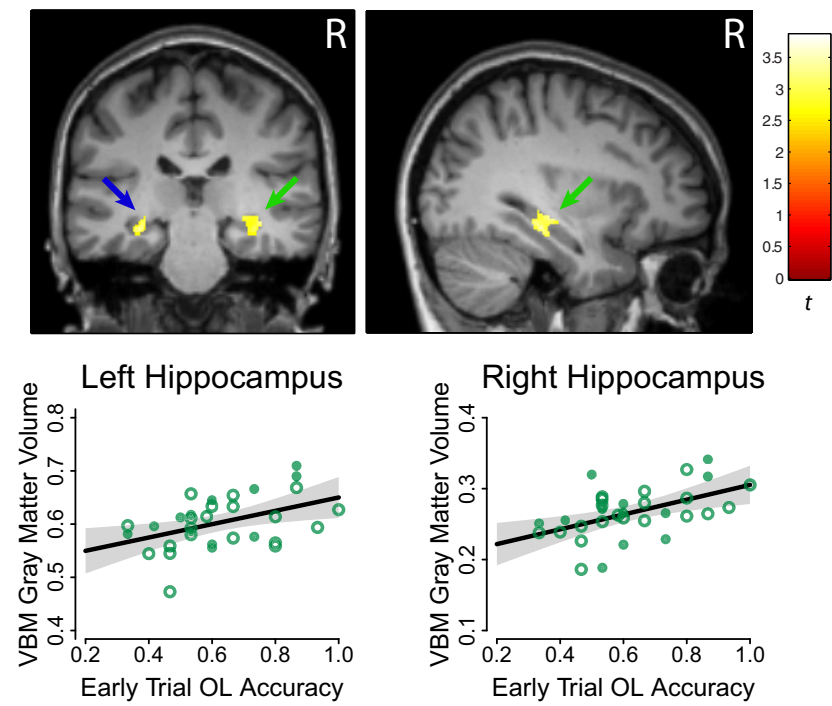

Figure 3. Gray matter volume correlated with context-dependent Critical Decision accuracy in new $0 \mathrm{~L}$ mazes. Green arrow denotes significant effect in the right hippocampus, blue arrow denotes effect in the left hippocampus. Results are thresholded at $p<0.01$. Scatter plots indicate the distribution of individual gray matter volume estimates around the regression line, solid dots indicating male participants, and open circles indicating female participants. Shaded regions depict uncorrected $95 \%$ confidence intervals. $R$, right hemisphere.

hippocampal effects did not extend into neighboring brain areas. No negative relationships with OL performance were observed.

Critically, these effects were specific to spatial disambiguation: there was no positive relationship between gray matter volume and NOL maze counterpart performance, even at a reduced voxelwise threshold of $p<0.05$. Results from subsequent analysis of the unified model demonstrated that variance in both left and right hippocampal volumes robustly correlated with OL accuracy, corrected for multiple comparisons, when holding learning rate constant (left/right: $x y z=-31,-24,-17 / 36,-31,-11$; $\left.t_{(25)}=3.99 / 5.02 ; p=0.0003 / 0.00002 ; k=339 / 618\right)$. Direct comparison of the relationship between volume and accuracy for the OL and NOL conditions confirmed that both left and right hippocampal volumes related significantly more positively to OL accuracy (left/right: $x y z=-31,-24,-15 / 33,-24,-17 ; t_{(25)}=$ 3.10/3.66; $p=0.002 / 0.001)$. The OL $>$ NOL difference was also significant for the right anterior cingulate $\left(x y z=12,47,1 ; t_{(25)}=\right.$ 3.69; $p=0.001)$.

Interestingly, we did observe negative relationships between prefrontal structural variability and NOL accuracy in the anterior cingulate cortex (left/right ACC: $x y z$ : $-12,42,10 / 6,30,-15$; $\left.t_{(28)}=4.31 / 3.91 ; p=0.00009 / 0.0002 ; k=1111 / 1395\right)$, orbitofrontal cortex (left/right OFC: xyz: $-18,41,-21 / 20,42,-23$; $\left.t_{(28)}=3.43 / 4.17 ; p=0.001 / 0.0001 ; k=278 / 266\right)$, dorsolateral PFC (left/right DLPFC: $x y z:-21,48,25 / 35,48,6 ; t_{(28)}=4.40 /$ $4.21 ; p=0.00007 / 0.0001 ; k=351 / 1169)$, and the ventrolateral PFC $\left(x y z:-51,39,13 ; t_{(28)}=4.80 ; p=0.00002 ; k=176\right)$. As determined by subsequent analysis of the unified model, voxels in the right anterior cingulate $\left(x y z: 6,33,22 ; t_{(28)}=3.46 ; p=0.001\right.$; $k=208)$ and right DLPFC $\left(x y z: 35,47,4 ; t_{(28)}=4.17 ; p=0.0002\right.$; $k=177)$ were negatively correlated with NOL Early task phase performance variance when holding learning rate constant. We found no significant relationships between caudate gray matter volume and accuracy in either condition.

\section{Prefrontal gray matter volume and learning rate}

Gray matter volume from a large region of left rostral DLPFC $\left(x y z:-31,57,18 ; t_{(28)}=3.84 ; p=0.0003 ; k=315\right)$ had a significant positive relationship with $d_{a}$ from the learning phase of the OL condition, indicating that individuals who rapidly reached peak context-dependent navigation performance across learning trials have greater gray matter volume estimates in the DLPFC (Fig. 4a). A negative relationship with $d_{a}$ was observed in the left amygdala $\left(x y z:-21,-6,-23 ; t_{(28)}=3.18 ; p=0.002 ; k=220\right)$, indicating larger amygdala gray matter volume correlates with slower learning (Fig. 4b).

There was no positive relationship between gray matter and NOL maze counterpart performance, even at a reduced voxelwise threshold of $p<0.05$. Direct comparison of the relationship between volume and learning rate for the OL and NOL conditions using the unified model demonstrated that left DLFPC volumes related significantly more positively to OL learning rate $\left(x y z=-39,50,3 ; t_{(25)}=3.11\right)$. Furthermore, variance in left DLPFC volumes significantly correlated with OL learning rate when holding average accuracy constant (but not vice versa; $\left.x y z=-36,48,13 ; t_{(25)}=3.24 ; p=0.002 ; k=169\right)$. Scatter plots exploring the specificity of our effects in the hippocampus and DLPFC revealed that the peak left and right hippocampal voxels correlating most strongly with OL accuracy (Fig. $5 a, b$ ) had only a weak relationship with OL learning rate (Fig. $5 d$,e), while the peak left DLPFC voxel that correlated significantly with OL learning rate (Fig. $5 f$ ) also showed a moderate relationship with accuracy (Fig. 5c). This trend is consistent both with the role of the DLPFC in decision making and a relationship between our accuracy and learning measures. However, our multiple regression analyses demonstrated that DLPFC volumes correlate with learning rate when holding accuracy constant, and when separately examining correlates of accuracy there was not a significant relationship with accuracy in this region even at our exploratory reduced cluster extent, which may be critical for inference of volumetric data. These data suggest that large-scale morphology in the MTL and PFC may be important, to different degrees, for partially distinct facets of spatial disambiguation.

While there were no positive relationships between gray matter and NOL learning rate, we did observe negative relationships with NOL learning rate in the anterior cingulate $(x y z:-6,32,21$; $\left.t_{(28)}=4.24 ; p=0.0001 ; k=1335\right)$, OFC $\left(x y z: 9,59,-26 ; t_{(28)}=\right.$ $3.83 ; p=0.0003 ; k=326)$, DLPFC $\left(x y z:-15,56,24 ; t_{(28)}=3.99\right.$; $p=0.0002 ; k=447)$, and ventrolateral PFC (xyz: 38, 54, -9; $\left.t_{(28)}=3.78 ; p=0.0004 ; k=262\right)$. While it is difficult to interpret negative relationships between anatomical variability and NOL accuracy and learning rate, these data suggest that morphology in prefrontal areas that support OL maze performance may impair NOL maze performance-potentially reflecting a bias away from stimulus-response learning that could support efficient navigation of unambiguous routes. Our findings do not suggest that neurons in our ROIs are inactive for NOL route navigation, but demonstrate that, in contrast to the OL condition, the ability to perform computations needed for NOL maze navigation does not systematically improve with additional neural resources in these areas.

Importantly, our disambiguation (OL)-specific correlates remain significant when local gray matter volume estimates are measured as "relative" rather than "absolute" volume (regressing out the influence of global gray matter volumes; Accuracy, left/ right hippocampus: $t_{(27)}=3.11 / 3.14$; anterior cingulate cortex: $t_{(27)}=3.59$. Learning rate, DLPFC: $t_{(27)}=4.84$; amygdala: $t_{(27)}=$ $-3.38)$. 


\section{Discussion}

Our experiment provides a novel examination of how individual differences in brain structure relate to contextdependent memory and navigational abilities. Our results provide evidence that accurate context-dependent overlapping route navigation during learning relates to individual differences in hippocampal gray matter volume in healthy young adults. Furthermore, how rapidly people are able to learn to navigate overlapping routes relates to gray matter volume differences in rostral DLPFC.

\section{Hippocampal gray matter volume and Critical Decision accuracy}

We hypothesized that greater volume in the hippocampus would support a more robust anatomical framework for the representation of contextual and spatial details in sequential navigational memories, and could provide additional neural resources for flexibly discriminating navigational representations based on context at retrieval. Our results support this hypothesis, demonstrating that individual differences in context-dependent navigation ability are correlated with gray matter volume in the hippocampal body. This finding is consistent with prior evidence that hippocampal activity supports learning and retrieval of overlapping sequential memories (Kumaran and Maguire, 2006; Ross et al., 2009; Brown et al., 2010, 2012; Brown and Stern, 2013). Furthermore, we found a relationship specific to the overlapping mazes between Critical Decision accuracy and gray matter volume within the anterior cingulate, which is a medial prefrontal structure intimately connected with the hippocampus and believed to be critical to behavioral and mnemonic flexibility (Barbas et al., 1999; Allman et al., 2001; Beckmann et al., 2009; Bunce and Barbas, 2011; Rushworth et al., 2011).

Our findings provide novel insight into the question of how hippocampal structure relates to flexible navigational abilities. Episodic memory is a key component of our task, and episodic and spatial memory are linked in the hippocampus (Eichenbaum et al., 1999; Eichenbaum, 2000; Burgess, 2002; Buzsáki, 2005; MacDonald et al., 2011). Hippocampal volumes have been linked with nonspatial memory performance in humans (Rodrigue et al., 2013), and support memory for context (Horner et al., 2012). Prior studies have also linked hippocampal structure to topographical memory and spatial strategies (Maguire et al., 2006; Bohbot et al., 2007; Iaria et al., 2008; Woollett and Maguire, 2011; Hartley and Harlow, 2012), and recent data (Schinazi et al., 2013) suggest

a

b is nonsignificant.
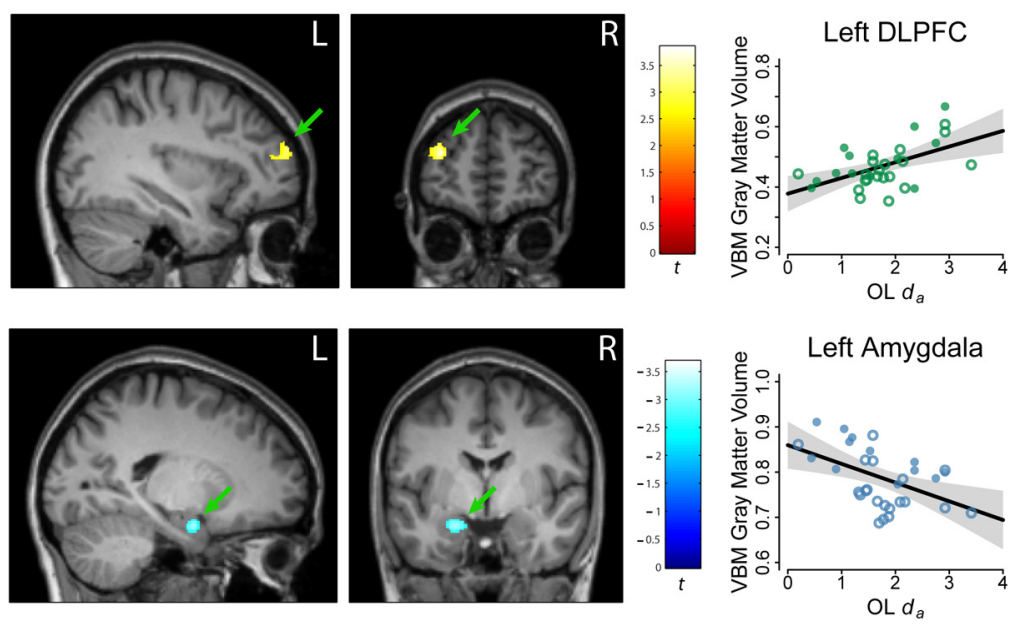

Figure 4. Gray matter volume correlated with Critical Decision learning rate $\left(d_{a}\right)$ of new $0 \mathrm{~L}$ mazes. $\boldsymbol{a}$. Green arrow denotes significant positive relationship in the left DLPFC. $\boldsymbol{b}$, Green arrow denotes significant negative relationship in the left amygdala. Results are thresholded at $p<0.01$. Scatter plots indicate the distribution of individual gray matter volume estimates around the regression line, solid dots indicating male participants, and open circles indicating female participants. Shaded regions indicate uncorrected $95 \%$ confidence intervals. R, right hemisphere; $L$, left hemisphere.
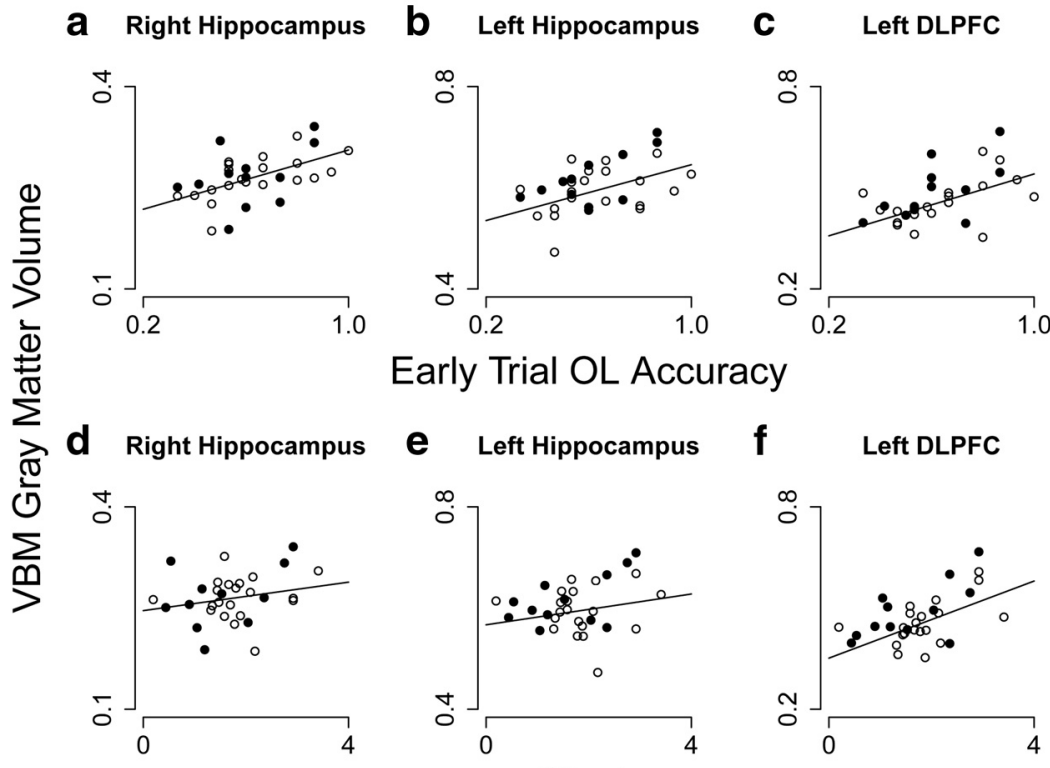

Early Trial OL Accuracy
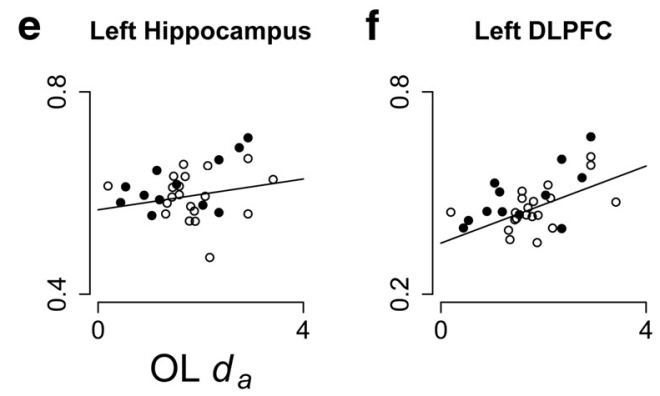

Figure 5. Scatter plots further characterizing the specificity of our structure-performance relationships in peak voxels in the hippocampus and DLPFC identified in our SPM analyses. Top row plots $(\boldsymbol{a}-\boldsymbol{c})$ indicate the distribution of individual gray matter volume estimates around the accuracy regression line. Bottom row plots $(\boldsymbol{d}-\boldsymbol{f})$ indicate the distribution of individual gray matter volume estimates around the learning rate $\left(d_{a}\right)$ regression line. Solid dots indicate male participants, and open circles indicate female participants. Right and left peak hippocampal voxels show a markedly stronger relationship with OL accuracy $(\boldsymbol{a}$ and $\boldsymbol{b})$ than OL learning rate ( $\boldsymbol{d}$ and $\boldsymbol{e}$ ). The peak left DLPFC voxel that correlated with $0 \mathrm{~L}$ learning rate $(\boldsymbol{f})$ also showed a moderate relationship with accuracy $(\boldsymbol{c})$, although the distribution of individual participants around these lines differs and the relationship with accuracy

larger hippocampal volume results in an increased ability to link navigational experiences with cognitive maps, and supports memory for the directional relationship between landmarks. Spatial reasoning could further support efficient use of existing route information to flexibly orient to landmarks in the environment and avoid navigational errors. Importantly, Early task phase performance in our study is influenced by learning, and fMRI research has demonstrated that hippocampal activity is particularly important for the learning of cognitive map informa- 
tion (Wolbers and Büchel, 2005; Sherrill et al, 2013) and flexible overlapping route memories (Brown and Stern, 2013). In our study, navigators who are efficient at distinguishing navigational episodes while also flexibly using contextual information and existing spatial knowledge can better avoid navigational errors when crossing paths with other navigational routes.

\section{Prefrontal gray matter volume and Critical Decision learning rate}

Our finding that gray matter in the rostral DLPFC had a significant positive relationship with learning rate is consistent with evidence suggesting rostral prefrontal activity supports both learning and retrieval of overlapping navigational routes (Brown et al., 2010; Brown and Stern, 2013). Furthermore, the hippocampal body (whose volumes correlate in this study with early $\mathrm{OL}$ accuracy) is functionally connected with rostral lateral PFC during overlapping route navigation (Brown et al., 2012). Lateral PFC has been implicated in cognitive control and decision making, with more rostral areas functioning on a more abstract or integrative level (Badre, 2008). Critically, DLPFC supports relational memory encoding (Murray and Ranganath, 2007; Qin et al., 2007; Blumenfeld et al., 2011). In the present study, greater gray matter volume in the DLPFC could provide greater neural resources for sequential route encoding and the efficient organization of recent experience and feedback into successful planning of context-dependent behavior on subsequent trials.

It is interesting to note that hippocampal and DLPFC volumes correlate strongly with Early task phase performance and learning rate, respectively, when variance from the other measure is held constant. Both learning-related measures are correlated, and as discussed above the results from our separate interrogations of these factors are consistent with a functional role for both regions in overlapping route performance. However, individuals with the steepest learning curve across trials were not necessarily the individuals who began and performed at the highest level of accuracy in the earliest trials of the task. When holding learning rate constant, results from the unified model suggest that neural resources in the hippocampus influence our ability to flexibly access and use previously encoded spatial information in support of accurate navigation during early trials, when interference from the learned behavioral responses of the other routes is highest. Neural resources in the DLPFC may be particularly important for the ability to incorporate recent feedback, perhaps maintained in working memory, into the integration of new response patterns with existing behavioral associations in the environment-a process that could be essential to overlapping route learning.

We observed an inverse relationship between gray matter volume in the left amygdala and overlapping maze learning rate. This result is surprising because amygdala function has typically been associated with the hippocampus and declarative memory (Fenker et al., 2005; Kensinger and Schacter, 2006). However, subregions of the amygdala have been implicated in conditioned responses, habit memory, and regulating different memory systems (Everitt and Robbins, 2005; Wingard and Packard, 2008; Lingawi and Balleine, 2012). Our findings may reflect structural differences that correspond to altered or disrupted balance between goal-directed and stimulus-response subcircuitry in the amygdala, such that participants with larger gray matter volume in the amygdala have difficulty overcoming previously learned and consolidated behavioral responses at the overlapping intersections. Prior anatomical research has linked decreased volumes in the anterior hippocampus to increased spatial expertise (Maguire et al., 2006), and it is possible that these adjacent regions of the
MTL relate to flexible navigation ability in a common manner. It will be of particular interest to examine this relationship further in future studies.

Importantly, hippocampal and DLPFC volumes were not positively correlated with NOL maze performance, even at reduced thresholds, and correlations with OL performance measures were significantly more positive than for NOL performance when directly compared. This suggests our results are not a general effect of route navigation ability, and indicate gray matter volumes in the hippocampus and PFC most directly support cognitive processes underlying flexible context-dependent navigation. Our findings support the prediction that the ability to perform computations needed for NOL maze navigation does not systematically improve with additional neural resources in these regions. The negative correlation between prefrontal volumes and NOL accuracy and learning is consistent with greater activity in these areas for accurate OL than accurate NOL navigation (Brown et al., 2010; Brown and Stern, 2013) and, importantly, suggests that morphology in these areas that facilitates flexible navigation may impair efficient learning of unambiguous response memories. These findings could have important clinical implications- our paradigm and results, targeting the intersection of episodic memory and navigation, could be used as a framework for research examining impaired flexible and goaldirected navigation in healthy and diseased aging (Lithfous et al., 2013).

\section{Conclusions}

We provide evidence that hippocampal volume estimates in healthy young adults relate to accuracy navigating contextdependent overlapping routes during early learning. Furthermore, gray matter volume in a rostral region of the DLPFC, known to functionally interact with the hippocampus in support of spatial disambiguation at retrieval, was significantly related to our measure of overlapping route learning rate. These results were specific to overlapping maze navigation, and were not accounted for by global gray matter volume differences. Together, our targeted analysis provides novel evidence that individual differences in gray matter volume in the hippocampus and PFC relate to our ability to rapidly learn and flexibly navigate routes that cross paths with existing navigational memories. These findings shed new light on the relationship between neuroanatomical structure and navigational and episodic memory processes in the healthy human brain.

\section{References}

Agster KL, Fortin NJ, Eichenbaum H (2002) The hippocampus and disambiguation of overlapping sequences. J Neurosci 22:5760-5768. Medline

Akaike H (1974) A new look at the statistical model identification. IEEE Trans Automat Contr 19:716-723. CrossRef

Allman JM, Hakeem A, Erwin JM, Nimchinsky E, Hof P (2001) The anterior cingulate cortex. The evolution of an interface between emotion and cognition. Ann NY Acad Sci 935:107-117. Medline

Ashburner J (2007) A fast diffeomorphic image registration algorithm. Neuroimage 38:95-113. CrossRef Medline

Badre D (2008) Cognitive control, hierarchy, and the rostro-caudal organization of the frontal lobes. Trends Cogn Sci 12:193-200. CrossRef Medline

Barbas H, Ghashghaei H, Dombrowski SM, Rempel-Clower NL (1999) Medial prefrontal cortices are unified by common connections with superior temporal cortices and distinguished by input from memory-related areas in the rhesus monkey. J Comp Neurol 410:343-367. CrossRef Medline

Beckmann M, Johansen-Berg H, Rushworth MF (2009) Connectivity-based parcellation of human cingulate cortex and its relation to functional specialization. J Neurosci 29:1175-1190. CrossRef Medline

Blumenfeld RS, Parks CM, Yonelinas AP, Ranganath C (2011) Putting the 
pieces together: the role of dorsolateral prefrontal cortex in relational memory encoding. J Cogn Neurosci 23:257-265. CrossRef Medline

Bohbot VD, Lerch J, Thorndycraft B, Iaria G, Zijdenbos AP (2007) Gray matter differences correlate with the spontaneous strategies in a human virtual navigation task. J Neurosci 27:10078-10083. CrossRef Medline

Brown TI, Stern CE (2013) Contributions of medial temporal lobe and striatal memory systems to learning and retrieving overlapping spatial memories. Cereb Cortex. Advance online publication. Retrieved Feb. 28, 2013. doi:10.1093/cercor/bht041. CrossRef

Brown TI, Ross RS, Keller JB, Hasselmo ME, Stern CE (2010) Which way was I going? Contextual retrieval supports the disambiguation of well learned overlapping navigational routes. J Neurosci 30:7414-7422. CrossRef Medline

Brown TI, Ross RS, Tobyne SM, Stern CE (2012) Cooperative interactions between hippocampal and striatal systems support flexible navigation. Neuroimage 60:1316-1330. CrossRef Medline

Bunce JG, Barbas H (2011) Prefrontal pathways target excitatory and inhibitory systems in memory-related medial temporal cortices. Neuroimage 55:1461-1474. CrossRef Medline

Burgess N (2002) The hippocampus, space, and viewpoints in episodic memory. Q J Exp Psychol A 55:1057-1080. CrossRef Medline

Buzsáki G (2005) Theta rhythm of navigation: link between path integration and landmark navigation, episodic and semantic memory. Hippocampus 15:827-840. CrossRef Medline

Eichenbaum H (2000) A cortical-hippocampal system for declarative memory. Nat Rev Neurosci 1:41-50. CrossRef Medline

Eichenbaum H, Dudchenko P, Wood E, Shapiro M, Tanila H (1999) The hippocampus, memory, and place cells: is it spatial memory or a memory space? Neuron 23:209-226. CrossRef Medline

Everitt BJ, Robbins TW (2005) Neural systems of reinforcement for drug addiction: from actions to habits to compulsion. Nat Neurosci 8:14811489. CrossRef Medline

Fenker DB, Schott BH, Richardson-Klavehn A, Heinze HJ, Düzel E (2005) Recapitulating emotional context: activity of amygdala, hippocampus and fusiform cortex during recollection and familiarity. Eur J Neurosci 21:1993-1999. CrossRef Medline

Ginther MR, Walsh DF, Ramus SJ (2011) Hippocampal neurons encode different episodes in an overlapping sequence of odors task. J Neurosci 31:2706-2711. CrossRef Medline

Hartley T, Harlow R (2012) An association between human hippocampal volume and topographical memory in healthy young adults. Front Hum Neurosci 6:338. Medline

Hasselmo ME, Eichenbaum H (2005) Hippocampal mechanisms for the context-dependent retrieval of episodes. Neural Netw 18:1172-1190. CrossRef Medline

Horner AJ, Gadian DG, Fuentemilla L, Jentschke S, Vargha-Khadem F, Duzel E (2012) A rapid, hippocampus-dependent, item-memory signal that initiates context memory in humans. Curr Biol 22:2369-2374. CrossRef Medline

Iaria G, Lanyon LJ, Fox CJ, Giaschi D, Barton JJ (2008) Navigational skills correlate with hippocampal fractional anisotropy in humans. Hippocampus 18:335-339. CrossRef Medline

Kensinger EA, Schacter DL (2006) Amygdala activity is associated with the successful encoding of item, but not source, information for positive and negative stimuli. J Neurosci 26:2564-2570. CrossRef Medline

Kumaran D, Maguire EA (2006) The dynamics of hippocampal activation during encoding of overlapping sequences. Neuron:617-629.

Lingawi NW, Balleine BW (2012) Amygdala central nucleus interacts with dorsolateral striatum to regulate the acquisition of habits. J Neurosci 32:1073-1081. CrossRef Medline

Lithfous S, Dufour A, Després O (2013) Spatial navigation in normal aging and the prodromal stage of Alzheimer's disease: insights from imaging and behavioral studies. Ageing Res Rev 12:201-213. CrossRef Medline

MacDonald CJ, Lepage KQ, Eden UT, Eichenbaum H (2011) Hippocampal "time cells" bridge the gap in memory for discontiguous events. Neuron 71:737-749. CrossRef Medline
Maguire EA, Woollett K, Spiers HJ (2006) London taxi drivers and bus drivers: a structural MRI and neuropsychological analysis. Hippocampus 16: 1091-1101. CrossRef Medline

Maldjian JA, Laurienti PJ, Burdette JH (2004) Precentral gyrus discrepancy in electronic versions of the Talairach atlas. Neuroimage 21:450-455. CrossRef Medline

Maldjian JA, Laurienti PJ, Kraft RA, Burdette JH (2003) An automated method for neuroanatomic and cytoarchitectonic atlas-based interrogation of fMRI data sets. Neuroimage 19:1233-1239. CrossRef Medline

Mechelli A, Price CJ, Friston KJ, Ashburner J (2005) Voxel-based morphometry applications of the human brain: methods and applications. Curr Med Imaging Rev 1:1-9. CrossRef

Murray LJ, Ranganath C (2007) The dorsolateral prefrontal cortex contributes to successful relational memory encoding. J Neurosci 27:5515-5522. CrossRef Medline

Obuchowski NA (2003) Receiver operating characteristic curves and their use in radiology. Radiology 229:3-8. CrossRef Medline

Qin S, Piekema C, Petersson KM, Han B, Luo J, Fernández G (2007) Probing the transformation of discontinuous associations into episodic memory: an event-related fMRI study. Neuroimage 38:212-222. CrossRef Medline

R Development Core Team (2012) R: a language and environment for statistical computing. Vienna, Austria: the R Foundation for Statistical Computing.

Rodrigue KM, Daugherty AM, Haacke EM, Raz N (2013) The role of hippocampal iron concentration and hippocampal volume in age-related differences in memory. Cereb Cortex 23:1533-1541. CrossRef Medline

Ross RS, Brown TI, Stern CE (2009) The retrieval of learned sequences engages the hippocampus: evidence from fMRI. Hippocampus 19:790-799. CrossRef Medline

Rushworth MF, Noonan MP, Boorman ED, Walton ME, Behrens TE (2011) Frontal cortex and reward-guided learning and decision-making. Neuron 70:1054-1069. CrossRef Medline

Schinazi VR, Nardi D, Newcombe NS, Shipley TF, Epstein RA (2013) Hippocampal size predicts rapid learning of a cognitive map in humans. Hippocampus 23:515-528. CrossRef Medline

Sherrill KR, Erdem UM, Ross RS, Brown TI, Hasselmo ME, Stern CE (2013) Hippocampus and retrosplenial cortex combine path integration signals for successful navigation. J Neurosci 33:19304-19313. CrossRef Medline

Smith DM, Mizumori SJ (2006) Learning-related development of contextspecific neuronal responses to places and events: the hippocampal role in context processing. J Neurosci 26:3154-3163. CrossRef Medline

Tzourio-Mazoyer N, Landeau B, Papathanassiou D, Crivello F, Etard O, Delcroix N, Mazoyer B, Joliot M (2002) Automated anatomical labeling of activations in SPM using a macroscopic anatomical parcellation of the MNI MRI single-subject brain. Neuroimage 15:273-289. CrossRef Medline

Wingard JC, Packard MG (2008) The amygdala and emotional modulation of competition between cognitive and habit memory. Behav Brain Res 193:126-131. CrossRef Medline

Wixted JT, Mickes L (2010) A continuous dual-process model of remember/know judgments. Psychol Rev 117:1025-1054. CrossRef Medline

Wolbers T, Büchel C (2005) Dissociable retrosplenial and hippocampal contributions to successful formation of survey representations. J Neurosci 25:3333-3340. CrossRef Medline

Wood ER, Dudchenko PA, Robitsek RJ, Eichenbaum H (2000) Hippocampal neurons encode information about different types of memory episodes occurring in the same location. Neuron 27:623-633. CrossRef Medline

Woollett K, Maguire EA (2011) Acquiring "the Knowledge" of London's layout drives structural brain changes. Curr Biol 21:2109-2114. CrossRef Medline

Zilli EA, Hasselmo ME (2008) Modeling the role of working memory and episodic memory in behavioral tasks. Hippocampus 18:193-209. CrossRef Medline 